\title{
ASPECTOS BIOLÓGICOS E TABELA DE VIDA DE FERTILIDADE DE APHIS GOSSYPII GLOVER (HEMIPTERA: APHIDIDAE) EM CULTIVARES DE ALGODOEIRO DELTAOPAL E NUOPAL
}

\author{
M. Funichello, L.L. Costa, A.C. Busoli \\ Universidade Estadual Paulista, Faculdade de Ciências Agrárias e Veterinárias, Departamento de Fitossani- \\ dade, Laboratório de Criação de Insetos, Via de Acesso Prof. Paulo Donato Castellane s/non, CEP 14884-900, \\ Jaboticabal, SP, Brasil. E-mail: mariagro2@gmail.com
}

\section{RESUMO}

Cultivares modificadas geneticamente podem afetar a biologia de organismos não-alvos e, com isso, modificar as relações tritróficas nos agroecossistemas. No caso de algodoeiro Bt, tem provocado alterações na biologia de Aphis gossypii Glover (Hemiptera: Aphididae) e influenciado a dinâmica populacional deste inseto no campo. O objetivo deste trabalho foi avaliar alguns aspectos biológicos do pulgão A. gossypii em folhas de cultivar de algodoeiro transgênico NuOPAL (Bollgard I) e de sua isolinha convencional DeltaOPAL. O experimento foi realizado em laboratório a $25 \pm 1^{\circ} \mathrm{C}$, umidade relativa de $70 \pm 10 \%$ e fotofase de 12 horas. Os tratamentos foram constituídos pelos cultivares de algodoeiro, com 40 repetições cada, utilizando-se o delineamento inteiramente casualizado. Cada parcela foi constituída por uma placa de Petri com tampa vazada e cobertas com "voil", contendo solução ágar-água a $1 \%$ solidificada, sobre a qual foi disposto um disco foliar de $6 \mathrm{~cm}$ de diâmetro, onde ninfas de um dia de idade foram individualizadas. As placas foram vistoriadas duas vezes ao dia avaliando-se duração dos estádios ninfais, fase ninfal total, períodos reprodutivo e pós-reprodutivo, número total de ninfas/fêmea e longevidade. Com os dados biológicos foram elaboradas tabela de vida de fertilidade do pulgão em cada cultivar. A duração dos estádios imaturos, longevidade de fêmeas, aspectos reprodutivos e de fertilidade de A. gossypii não foram influenciados pelos cultivares de algodoeiro.

PALAVRAS-CHAVE: Pulgão do algodoeiro, biologia, Gossypium hirsutum, Bollgard I, Cry1Ac.

\begin{abstract}
BIOLOGICAL ASPECTS AND FERTILITY LIFE TABLE OF APHIS GOSSYPII GLOVER (HEMIPTERA: APHIDIDAE) ON COTTON CULTIVARS DELTAOPAL AND NUOPAL. Genetically modified plants can affect the biology of non-targeted organisms, and change the tritrofic relations in the agro-ecosystem. Bt cotton cultivars have led to changes in the biology of A phis gossypii Glover (Hemiptera: Aphididae), and have influenced the population dynamics of this insect in the field. The objective of the present study was to assess some biological parameters of A. gossypii in genetically modified cotton NuOPAL (Bollgard I) and DeltaOPAL cultivars. The experiment was carried out under controlled conditions, at $25 \pm 1^{\circ} \mathrm{C}, 70 \pm 10 \%$ relative humidity, and a 12-hour photophase. The experiment was installed in a completely randomized design, with DeltaOPAL and NuOPAL (Bollgard I) cultivars considered treatments, and 40 replications. Leaf circles of 6 $\mathrm{cm}$ in diameter were placed into Petri dishes containing $1 \%$ solidified agar-water, and nymphs of up to one-day-old were placed individually on these circles. The evaluations were carried out on a daily basis, and the biological parameters evaluated were: duration of each nymph stage and total phase, duration of reproductive and post-reproductive periods, total nymphs/females, and longevity. The parameters of the fertility life table were also assessed. The duration of nymph states and nymph stages, duration of reproductive and post-reproductive periods, longevity, fertility and total number of nymphs per female did not present any significant differences. The genetically modified NuOPAL does not affect the biology of the cotton aphid A. gossypii.
\end{abstract}

KEY WORDS: Cotton aphid, biology, Gossypium hirsutum, Bollgard I, Cry1Ac. 


\section{INTRODUÇÃO}

O agroecossistema algodoeiro apresenta grande diversidade de artrópodes-pragas, assim como de inimigos naturais, que tem grande importância na regulação das populações, tornando seu cultivo sustentável (Busoli et al., 2008). Dentre as pragas que podem causar dano econômico à cultura, pode-se mencionar Aphis gossypii Glover, 1877 (Hemiptera: Aphididae), um dos primeiros insetos que surgem logo após a emergência das plantas, provocando danos diretos pela contínua sucção de seiva, afetando o crescimento das plantas e, danos indiretos, pela transmissão do vírus do vermelhão e do mosaico das nervuras (MichelotTo; Busoli, 2003a; Michelotto et al., 2003).

Aphis gossypiiécosmopolita, encontrando-se associado às diversas culturas de importância econômica sendo capaz de transmitir mais de 50 espécies de vírus (PeÑa-Martinez, 1992; Soglia et al., 2002). Esse afídeo coloniza o algodoeiro desde a fase de plântula, se reproduz de forma rápida e abundante, tendo seu desenvolvimento, sobrevivência e fecundidade afetados por fatores como temperatura e planta hospedeira (XIA et al., 1999; LiU et al., 2005).

A biologia de A. gossypii foi estudada em diversos hospedeiros como aboboreira, quiabeiro, pepino, meloeiro, crisântemo e algodoeiro por KHALIFA; ELDin (1964), Vendramim; NaKano (1981), KocoureK et al. (1994), SteENIS; EL- KHAWASS (1995), KeRSTING et al. (1999), Soglia et al. (2002) e Michelotto; Busoli (2003b), obtendo resultados semelhantes dos diversos parâmetros biológicos, comprovando por esses resultados tratar-se de uma praga altamente polífaga.

Pesquisas em biotecnologia têm proporcionado a obtenção de plantas geneticamente modificadas que expressam a proteína tóxica Cry1Ac (BовROwskI et al., 2003). Essas plantas podem controlar algumas pragas-alvos e reduzir a aplicação de inseticidas. Por meio desta tecnologia, foi obtido o algodão denominado Bollgard I, que produz em suas células a a-endotoxina de Bacillus thuringiensis var. kurstaki (Berliner, 1915) altamente patogênica para lagartas (RAMIRO; FARIA, 2006).

Pesquisas na China têm determinado o efeito desses cultivares sobre o pulgão $A$. gossypii, demonstrando maiores populações desse hemíptero em áreas de algodão $\mathrm{Bt}$, do que em áreas convencionais (DENG et al., 2003) ou até mesmo a não influência desses cultivares sobre as suas populações (Wu; Guo, 2003). Nesse mesmo país, Liv et al. (2005) verificaram que $A$. gossypii, alimentado por três gerações em algodão Bt, apresentou maior capacidade reprodutiva e maiores taxas de sobrevivência em comparação com pulgões alimentados com algodão não-Bt, com possível impacto sobre a dinâmica populacional da espécie. No Brasil, SujII et al. (2008) relataram que plantas de algodoeiro $\mathrm{Bt}$, que expressam proteínas tóxicas para uma praga-alvo, podem afetar a bionomia e a dinâmica das interações ecológicas no agroecossistema, devido a efeitos pleiotrópicos causados pela transgenia, tornando uma praga não-alvo importante. No entanto, esses autores observaram que os cultivares DP404 BG e a isolinha convencional DP 4049 não influenciaram o ciclo de vida de $A$. gossypii.

O objetivo deste trabalho foi avaliar os parâmetros biológicos de $A$. gossypii criado em folhas das cultivares DeltaOPAL e NuOPAL (Bollgard I), para confeccionar tabelas de vida de fertilidade e, com os resultados, avaliar os efeitos do evento Bollgard I (Cry1Ac) sobre alguns parâmetros biológicos do inseto, além de, em caso positivo, sugerir estudos mais detalhados sobre o impacto destas alterações no ciclo biológico deste inseto no campo, assim como as implicações destas alterações na biologia de seus predadores e parasitoides em condições de campo e de laboratório.

\section{MATERIAL E MÉTODOS}

\section{Obtenção de plantas e populações de Aphis gossypii}

As folhas utilizadas nos experimentos em laboratório foram obtidas utilizando-se 20 plantas dos cultivares dealgodoeiro DeltaOPALeNuOPAL (Bollgard I), semeadas em vasos plásticos de $5 \mathrm{~L}$, contendo terra, areia e esterco na proporção de 2:1:1. Cada vaso continha uma planta de cada cultivar, mantidas em uma casa de vegetação revestida com tela antiafídeo, com cobertura de filme plástico e com sombrite $50 \%$, até que as plantas atingissem 20 a 30 dias de idade. A cada 15 dias novos vasos foram semeados, de modo a possibilitar a constante produção de folhas novas para os experimentos, sempre que necessário.

De modo análogo e em outra casa de vegetação, os cultivares dealgodoeiro DeltaOPALeNuOPALforam semeados em vasos e colocadas no interior de gaiolas de estrutura metálica revestida com tela anti-afídeo (60 cm de diâmetro x $120 \mathrm{~cm}$ de altura). Aos 20 a 30 dias de idade, estas plantas foram infestadas com $A$. gossypii coletados no campo na cultivar de algodoeiro FMT701. Os pulgõesforam transferidos para as plantas com auxílio de um pincel de cerda macia número zero.

Ensaios para obtenção dos parâmetros biológicos de Aphis gossypii nos dois cultivares

Pulgões A. gossypii na fase reprodutiva foram coletadosem folhas doscultivares DeltaOPALeNuOPAL mantidas em vasos, como descrito anteriormente, para serem usados nos ensaios de laboratório. Foram preparados dois recipientes para cada cultivar, destinados à manutenção dos adultos, que consistiram 
de placas de Petri $(6,0 \mathrm{~cm}$ de diâmetro) contendo 15 $\mathrm{mL}$ de solução solidificada de ágar-água a $1 \%$ e um disco foliar de $6,0 \mathrm{~cm}$ de diâmetro de cada cultivar disposto no centro da placa, segundo metodologia de Michelotto; Busoli (2003b). Estes discos foram obtidos com auxílio de um vazador metálico de 6,0 $\mathrm{cm}$ de diâmetro, utilizando-se folhas dos cultivares de algodoeiro, previamente lavadas em água corrente e posteriormente secas em papel absorvente.

As placas contendo os adultos em fase reprodutiva foram vistoriadas três vezes ao dia para a coleta de ninfas, as quais foram utilizadas para o estudo da biologia nos doiscultivares dealgodão.Para a obtenção dessas ninfas demesmaidade, foram transferidos, com o auxílio de um pincel, quinze adultos ápteros de $A$. gossypii para discosfoliares doscultivares dealgodoeiro em cada placa (duas placas/cultivar). A tampa dessas placas continha uma abertura de $3 \mathrm{~cm}$ de diâmetro, coberta com uma tela antiafídeo para permitira aeração e evitar a fuga dos insetos. As placas foram mantidas em câmara climatizada, sob temperatura de $25 \pm 1^{\circ} \mathrm{C}$, umidade relativa de $70 \pm 10 \%$ e fotofase de 12 horas, para a produção constante e diária de ninfas de idade conhecida, ou seja, de até um dia de idade.

Com isto, para cada cultivar foram utilizadas quarenta placas de Petri contendo solução solidificada de ágar-água e um disco foliar de $6,0 \mathrm{~cm}$ de diâmetro, utilizadas para o confinamento de uma ninfa/placa de até um dia de idade e mantidas nas mesmas condições já citadas anteriormente.

As avaliações foram realizadas duas vezes ao dia e, a cada três a quatro dias, as placas contendo os discos foram trocadas, transferindo, com auxílio de um pincel, as respectivas ninfas para outras placas com discos foliares túrgidos. Na fase de ninfa determinaram-se as durações dos estádios e da fase ninfal. Durante a fase adulta avaliaram-se as durações dos períodos reprodutivo e pós-reprodutivo; longevidade; produção média diária e total de ninfas por fêmea.

Foiconsiderado como início da fase adulta, a partir do dia da produção da primeira ninfa, considerandose o período reprodutivo os dias em que cada fêmea produzisse ninfas e, o período pós-reprodutivo, a partir do dia que parassem de produzir ninfas até o dia de sua morte. A produção média diária de ninfas foi anotada, e estas retiradas das placas diariamente.

O delineamento experimental foi inteiramente casualizado, sendo os tratamentos constituídos pelos dois cultivares, com 40 repetições cada. Os dados obtidos (parâmetros biológicos) foram submetidos à análise de variância (Teste F) a 5\% de probabilidade. As análises estatísticas foram executadas pelo programa Estat/UNESP, do Departamento de Ciências Exatas da FCAV/UNESP/Campus de Jaboticabal.

Os parâmetros biológicos de $A$. gossypii determinados sobre os dois cultivares foram utilizados para elaborar a tabela de vida de fertilidade, segundo Silveira Neto et al. (1976) e Godor; Cividanes (2002). Por meio dos valores de intervalos de idade $(\mathrm{x})$, fertilidade específica $\left(\mathrm{m}_{\mathrm{x}}\right)$ e probabilidade de sobrevivência $\left(1_{x}\right)$ das tabelas de vida de fertilidade, foram calculadas a taxa líquida de reprodução $\left(R_{\mathrm{o}}\right)$, intervalo de tempoentre cada geração( $\mathrm{T})$, capacidade inata de aumentar em número $\left(\mathrm{r}_{\mathrm{m}}\right)$, razão finita de aumento (l), definida como o número de vezes que a população multiplica em uma unidade de tempo, e o tempo necessário para a população duplicar em número de indivíduos (TD), em que:

$\mathrm{R}_{\mathrm{o}}=\sum(\mathrm{mx} . \mathrm{lx}) \quad \mathrm{T}=\left(\sum \mathrm{mx} .1 \mathrm{x} . \mathrm{x}\right) /\left(\sum \mathrm{mx} .1 \mathrm{x}\right)$ $\mathrm{r}_{\mathrm{m}}=\log \mathrm{R}_{\mathrm{o}} /$ T. 0,4343 $\lambda=\operatorname{anti} \log \left(\mathrm{r}_{\mathrm{m}} \cdot 0,4343\right)$ $\mathrm{TD}=\mathrm{Ln}(2) / \mathrm{r}_{\mathrm{m}}$

\section{RESULTADOS E DISCUSSÃO}

\section{Parâmetros biológicos da fase ninfal}

Oscultivares dealgodoeironão afetaram a duração das fases de desenvolvimento de A. gossypii (Tabela 1). A fase ninfal durou $5,16 \pm 0,21$ e 5,06 $\pm 0,12$ dias quando $A$. gossypii foi criado sobre discos foliares dos cultivares DeltaOPAL e NuOPAL, respectivamente, não diferindo significativamente entre si (Tabela 1). Esses resultados confirmam os estudos de SujII et al. (2008) sobre A. gossypii e algodão Bt em casa de vegetação, onde o desenvolvimento das ninfas desse afídeo não foi afetado pela presença da proteína Cry1Ac. Resultado inferior para a duração da fase ninfal foi obtido por MichelotTo; Busoli (2003b), que obtiveram duração de 4,45 $\pm 0,75$ dias para a fase ninfal, nocultivar DeltaOPAL em câmara climatizada.

Tabela 1 - Duração média em dias ( \pm EP) das fases de desenvolvimento de Aphis gossypii. Temperatura de $25 \pm 1^{\circ} \mathrm{C}, \mathrm{UR}$ de $70 \pm 10 \%$, fotofase de $12 \mathrm{~h}$.

\begin{tabular}{lccccc}
\hline \multirow{2}{*}{ Cultivar } & \multicolumn{4}{c}{ Estádios ninfais } & \multirow{2}{*}{ Fase ninfal } \\
\cline { 2 - 5 } & $1^{\mathrm{o}}$ estádio & $2^{\circ}$ estádio & $3^{\circ}$ estádio & $4^{\mathrm{o}}$ estádio & \\
\hline DeltaOPAL & $1,66 \pm 0,12 \mathrm{a}$ & $1,18 \pm 0,10 \mathrm{a}$ & $1,20 \pm 0,09 \mathrm{a}$ & $1,11 \pm 0,07 \mathrm{a}$ & $5,16 \pm 0,21 \mathrm{a}$ \\
NuOPAL & $1,55 \pm 0,15 \mathrm{a}$ & $1,25 \pm 0,11 \mathrm{a}$ & $1,21 \pm 0,11 \mathrm{a}$ & $1,10 \pm 0,08 \mathrm{a}$ & $5,06 \pm 0,12 \mathrm{a}$ \\
\hline Teste F & $0,33^{\text {ns }}$ & $0,18^{\mathrm{ns}}$ & $0,01^{\mathrm{ns}}$ & $0,02^{\mathrm{ns}}$ & $0,16^{\mathrm{ns}}$ \\
CV $(\%)$ & 49,22 & 49,59 & 47,54 & 39,37 & 18,69 \\
\hline
\end{tabular}

Médias seguidas pela mesma letra na coluna não diferem significativamente entre si pelo teste de $\mathrm{F}(\mathrm{p} \leq 0,05)$. $\mathrm{EP}=\mathrm{erro}$ padrão da média. $\mathrm{n}=$ número de indivíduos avaliados. 
Resultados semelhantes dessas durações em dias para a fase ninfal foram encontrados por VENDRAMIM; NAKANO (1981), com 5,58 e 5,91 dias para ninfas de coloração amarelo e verde, respectivamente, porém criadas em plantas da variedade IAC- 17.

Estes resultados obtidos no presente ensaio são semelhantes aos obtidos por SujII et al. (2008), que estudaram os efeitos da toxina Cry1Ac na bionomia de A. gossypii, uma espécie-praga não alvo do cultivar Bt. Verificaram que não houve influência do cultivar $\mathrm{Bt}$, pois não observaram diferenças significativas entre os parâmetros biológicos obtidos nos cultivares, quanto à capacidade de desenvolvimento do inseto, porém, nada se pode afirmar se esses pulgões criados em plantas Bt forem utilizados posteriormente como presas, para a criação de predadores.

\section{Parâmetros biológicos da fase adulta}

Os adultos de $A$. gossypii não apresentaram diferença significativa entre os cultivares para as durações do período reprodutivo, pós-reprodutivo e longevidade (Tabela 2), demonstrando que a presença da proteína Cry1Ac na planta em que o pulgão sealimentava provavelmente não tenha influenciado a biologia da fase adulta. Operíodo reprodutivo desse inseto quando criado em discos foliares de plantas DeltaOPAL foi de 15,53 $\pm 0,91$ dias e de 13,46 $\pm 0,65$ dias quando mantido em discos foliares do cultivar $\mathrm{NuOPAL}$. Estes resultados corroboram SujII et al. (2008), que também não observaram efeito negativo na biologia da fase adulta do pulgão, mantido em casa de vegetação, porém, encontrou valores superiores do período reprodutivo, 16,35 $\pm 5,91$ e 16,18 \pm 6,66 para as cultivares DP 404 BG (Bollgard I) e sua isolinha DP 4049, respectivamente. Em relação ao período reprodutivo, pós-reprodutivo elongevidade correspondendo a $15,52 \pm 0,93,8,61 \pm 1,36$ e $24,33 \pm$ 1,59 , respectivamente, os resultados obtidos nessa pesquisa foram similares aqueles encontrados por
Michelotto; Busoli (2003b) para os adultos de $A$. gossypii criados de forma semelhante no cultivar DeltaOPAL.

A fecundidade média diária de fêmeas foi maior na cultivar transgênica NuOPAL, com 5,03 $\pm 0,31$ dias, enquanto no cultivar DeltaOPAL constataram-se 4,09 $\pm 0,21$ dias (Tabela 2). Entretanto, o número médio total de ninfas de $63,56 \pm 2,79$ no cultivar NuOPAL não diferiu significativamente com o cultivar DeltaOPAL, mesmo tendo sido inferior dois dias no cultivar Bt. Os resultados obtidos nos dois cultivares analisadas foram semelhantes aos obtidos por Michelotto; Busoli (2003b) no cultivar DeltaOPAL e maiores que os resultados de Liu et al. (2005) e SujII et al. (2008), que obtiveram menor fecundidade média total, com aproximadamente 47 ninfas por fêmea, para um período reprodutivo de 16,35 $\pm 5,91$ dias, em casa de vegetação, porém, ambos ensaios com outros cultivares de algodoeiro.

Os parâmetros da tabela de vida de fertilidade mostraram-se semelhantes quando A. gossypii foi criado sobre folhas dos dois cultivares de algodoeiro. O intervalo de tempo para cada geração (T) foi de 11,31 dias/geração para o cultivar NuOPAL, e 11,50 dias/geração para a DeltaOPAL. A taxa líquida de reprodução no cultivar transgênico foi 50,51 descendentes/fêmea, enquanto no cultivar não-Bt foi de 42,14 descendentes/fêmea, evidenciando que a proteína Cry1Ac não influenciou a reprodução do pulgão. Da mesma forma, a capacidade inata de aumentar em número foi de $\mathrm{r}_{\mathrm{m}}=0,3480$ para $\mathrm{o}$ cultivar geneticamente modificado e semelhante aquela observada para a sua isolinha, com um $r_{m}=$ 0,3262 . Também esses resultados foram semelhantes aos encontrados por SujII et al. (2008) com pulgões criados em cultivar que expressa a proteína tóxica Cry1Ac (DP 404 BG), em casa de vegetação, onde adultos apresentaram um $r_{m}=0,3711$, enquanto em plantas não-Bt o valor foi $r_{m}=0,3841$.

Tabela 2 - Duração média ( \pm EP) em dias dos parâmetros reprodutivos, longevidade e fecundidade de A. gossypii. Temperatura de $25 \pm 1^{\circ} \mathrm{C}$, UR de $70 \pm 10 \%$, fotofase de $12 \mathrm{~h}$.

\begin{tabular}{|c|c|c|c|}
\hline \multicolumn{4}{|c|}{ Períodos } \\
\hline Cultivar & Reprodutivo & Pós-reprodutivo & Longevidade \\
\hline DeltaOPAL & $15,53 \pm 0,91 \mathrm{a}$ & $8,43 \pm 0,97 a$ & $24,40 \pm 0,95 a$ \\
\hline NuOPAL & $13,46 \pm 0,65 a$ & $8,36 \pm 0,97 \mathrm{a}$ & $21,83 \pm 1,11 \mathrm{a}$ \\
\hline Teste F & $3,35^{\mathrm{ns}}$ & $0,00^{\mathrm{ns}}$ & $3,04^{\mathrm{ns}}$ \\
\hline $\mathrm{CV}(\%)$ & 30,16 & 63,85 & 24,65 \\
\hline \multicolumn{4}{|c|}{ Fecundidade (Número de ninfas/fêmea) } \\
\hline Cultivar & & & Diária \\
\hline DeltaOPAL & & & $4,09 \pm 0,21 b$ \\
\hline NuOPAL & & & $5,03 \pm 0,31 \mathrm{a}$ \\
\hline Teste F & & & $6,10^{*}$ \\
\hline $\mathrm{CV}(\%)$ & & & 32,24 \\
\hline
\end{tabular}

Médias seguidas pela mesma letra na coluna não diferem significativamente entre si pelo teste de Tukey $(\mathrm{p} \leq 0,05)$. EP = erro padrão da média. $\mathrm{n}$ = número de indivíduos avaliados. 


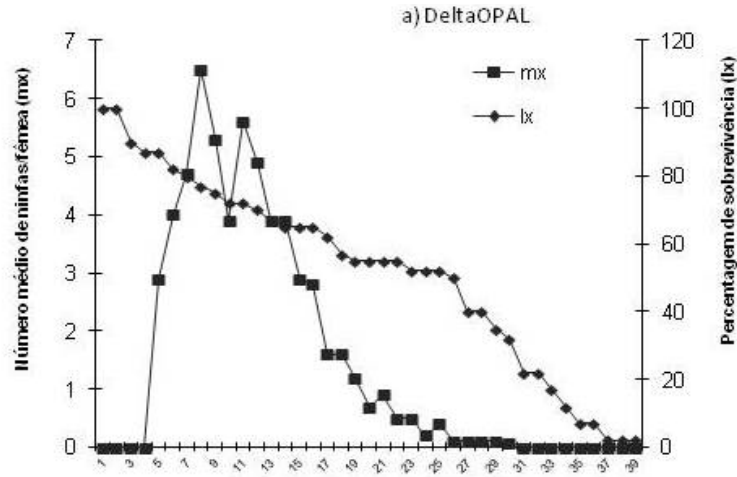

$\operatorname{Dias}(\mathrm{x})$

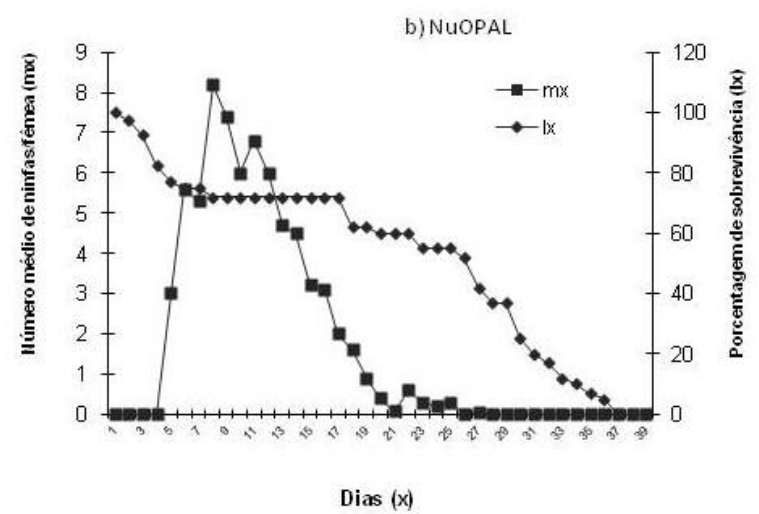

Fig. 1 - Número médio de ninfas/fêmea $\left(m_{x}\right)$ e percentagem de sobrevivência $\left(1_{x}\right)$ de Aphis gossypii mantidos sobre os cultivares NuOPAL e DeltaOPAL.

Amesmatendênciafoiobservada paraa razãofinita deaumento $(\lambda)$ sendoencontrados valores de $1,39 \mathrm{e} 1,42$, respectivamente, para DeltaOPAL e NuOPAL, sendo semelhanteaos resultados observados por MiснЕLOTTO; Busoli (2003b) para o pulgão criado nocultivar DeltaOPAL.Otemponecessário para a população duplicarem número de indivíduos (TD) foi de 2,16 dias e 1,99 dias, para DeltaOPAL e NuOPAL, respectivamente, o que mostra que o cultivar Bt não apresenta ação deletéria também sobre o desempenho dos adultos.

$\mathrm{O}$ período de maior fertilidade específica $\left(\mathrm{m}_{\chi}\right)$ para fêmeas mantidas nos cultivares DeltaOPAL e NuOPAL ocorreu entre o $6^{0}$ e $16^{0}$ dia, e com pico de fertilidade entre o $8^{\circ}$ e 9o dia, produzindo em média 6,50 ninfas por fêmea no cultivar DeltaOPAL (Fig. 1a) e um valor maior de 8,2 ninfas/fêmea no cultivar NuOPAL (Fig. 1b).

No geral, verifica-se que o cultivar transgênico $\mathrm{NuOPAL}$, não afetou significativamente o desenvolvimento das ninfas e nem o desempenho das fêmeas da espécie não-alvo $A$. gossypii, a exemplo de outros trabalhos (SujI et al., 2008) com outros cultivares transgênicos de algodoeiro no Brasil.

\section{CONCLUSÕES}

A duração dos instares e da fase ninfal, a duração dos períodos reprodutivo e pós-reprodutivo, a fecundidade e a longevidade de A. gossypii não são afetadas pelo cultivar transgênico NuOPAL.

O cultivar transgênico também não afeta a capacidade reprodutiva de $A$. gossypii, a duração de cada geração, a taxa líquida de reprodução, como também a capacidadeinata deaumentar emnúmero a população.

\section{AGRADECIMENTOS}

AoConselhoNacionaldeDesenvolvimentoCientífico eTecnológico-CNPq, pelaconcessãodebolsaaosautores.

\section{REFERENCIAS}

BOBROWSKI, V.L.; FIUZA, L.M.; PASQUALI, G.; ZANETTINI, M.H.B. Genes de Baccilus thuringiensis: uma estratégia para conferir resistência a insetos em plantas. Ciência Rural, v.34, n.1, p.843-850, 2003.

BUSOLI, A.C.; NAIS, J.; ARAUJO, C.R.; SILVA, E.A.; FUNICHELLO, M.; MICHELOTTO, M.D.; GUERREIRO, J.C. Atualidades sobre táticas e estratégias em MIP-algodoeiro. In: ARAUJO, E.S.;VACARI, A.M.; CARVALHO, J.S.; GOULART, R.M.; CAMPOS, A.P.; VOLPE, H.X.L. (Ed.). Tópicos em entomologia agrícola. Ribeirão Preto: Maxicolor Gráfica e Editora, 2008. p.39-54.

DENG, S.D.; XU, J.; ZHANG, Q.W.; ZHOU, S.W.; XU, G.J. Effect of transgenic Bacillus thuringiensis cotton on population dynamics of non-target pests and natural enemies. Acta Entomologica Sinica, v.46, p.1-5, 2003.

GODOY, K.B.; CIVIDANES, F.J. Tabelas de esperança de vida e fertilidade para Lipaphis erysimi (Kalt.) (Hemiptera: Aphididae) em condições de laboratório e campo. Neotropical Entomology, v. 31, n.1, p. 41-48, 2002.

KERSTING, U.; SATAR, S.; UYGUN, N. Effect of temperature on development rate and fecundity of apterous Aphis gossypii Glover (Homoptera: Aphididae) reared on Gossypium hirsutum L. Journal of Applied Entomology, v.123, p.23-27, 1999.

KHALIFA, A.; EL-DIN, S. Biological and ecological study on Aphis gossypii Glover (Hemiptera: Aphididae). Bulletin the la Societe Entomologique d'Egypte, v.48, p.131153, 1964.

KOCOUREK, F.; HARELKA, J.; BERÁNKOVA, J.; JAROSIK, V. Effects of temperature on development rate and intrinsic rate of increase of Aphis gossypii reared on greenhouse cucumbers. Entomologia Experimental et Applicata, v.71, n.1, p.59- 64, 1994.

LIU, X.D.; ZHAI, B.P.; ZHANG, X.X.; ZONG, J.M. Impact of transgenic cotton plants on a non-targed pest, 
Aphis gossypii Glover. Ecological Entomology, v. 30, p.307315, 2005.

MICHELOTTO, M.D.; BUSOLI, A.C. Eficiência de ninfas e adultos de Aphis gossypii Glover na transmissão do vírus do mosaico das nervuras do algodoeiro. Bragantia, v.62, n.2, p. 255-259, 2003a.

MICHELOTTO, M.D.; BUSOLI, A.C. Aspectos biológicos de Aphis gossypii Glover 1877 (Hemiptera: Aphididae) em três cultivares de algodoeiro e em três espécies de plantas daninhas. Ciência Rural, v.33, n.6, p. 99-104, $2003 b$.

MICHELOTTO, M.D.; SILVA, R.A. da; BUSOLI, A.C. Tabelas de esperança de vida e de fertilidade para Aphis gossypii Glover, 1887 (Hemiptera: Aphididae) em três cultivares de algodoeiro. Boletin de Sanidad Vegetal, v. 29, p.331-337, 2003.

PEÑA-MARTINEZ, R. Identificación de afidos de importância agricola. In: URIAS-M, C; RODRÍGUES-M, R.; ALEJANDRE-A, T. (Ed.). Áfidos como vectores de virus em México. 2.ed. México : Centro de Fitopatologia, 1992. p.1-135.

RAMIRO, Z.A.; FARIA, A.M. Levantamento de insetos predadores nos cultivares de algodão Bollgard DP 90 e convencional DeltaPine Acala 90. Arquivos do Instituto Biológico, São Paulo, v.73, n.1, p.119-121, 2006

SILVEIRA NETO, S.; NAKANO, O.; BALDIN, D.; VILLANOVA, N.A. Manual de ecologia dos insetos. São Paulo: Agronômica Ceres, 1976. 419p.
SOGLIA, M.C.M.; BUENO, V.H.P.; SAMPAIO, M.V. Desenvolvimento e sobrevivência de Aphis gossypii Glover, 1877 (Hemiptera: Aphididae) em diferentes temperaturas e cultivares comerciais de crisântemo. Neotropical Entomology, v.31, n.2, p.211-216, 2002.

SUJII, E.R.; TOGNI, P.H.B.; NAKASU, E.Y.T.; PIRES, C.S.S.; PAULA, D.P.; FONTES, E.M.G. Impacto de algodoeiro Bt na dinâmica populacional do pulgão-doalgodoeiro em casa de vegetação. Pesquisa Agropecuária Brasileira, v.43, n.10, p.1251-1256, 2008.

STEENIS, M. J. van; EL-KHAWASS, K.A.M.H. Life history of Aphis gossypii on cucumber: influence of temperature, host plant and parasitism. Entomologia Experimentalis et Applicata, v.76, p.121-131, 1995.

VENDRAMIM, J.D.; NAKANO, O. Aspectos biológicos de Aphis gossypii Glover, 1877 (Homoptera: Aphididae) em algodoeiro. Anais da Sociedade Entomológica do Brasil, v.10, n.2, p.163-173, 1981.

WU, K.; GUO, Y. Influences of Bacillus thurigiensis Berliner cotton planting on population dynamics of cotton aphid, Aphis gossypii Glover, in Northern China. Environmental Entomology, v.32, p.312-318, 2003.

XIA, J.Y.; VAN DER WERF, W.; RABBINGE, R. Influence of temperature on bionomics of cotton aphid Aphis gossypii on cotton. Entomologia Experimentalis et Applicata, v.90, p.25-35, 1999.

Recebido em 27/8/10

Aceito em 13/11/11 